\title{
Estimating the Fraction of Severe Malaria among Malaria-Positive Children: Analysis of Household Surveys in 19 Malaria-Endemic Countries in Africa
}

\author{
Cameron Taylor, ${ }^{1 \star}$ Sorrel M. L. Namaste, ${ }^{1}$ Joanna Lowell, ${ }^{2}$ Johanna Useem, ${ }^{1}$ and Yazoumé Yé ${ }^{3}$ \\ ${ }^{1}$ The DHS Program, ICF, Rockville, Maryland; ${ }^{2}$ The DHS Program, Vysnova Partners, Landover, Maryland; ${ }^{3}$ PMI Measure Malaria, \\ ICF, Rockville, Maryland
}

\begin{abstract}
To date, the only robust estimates of severe malaria cases include children who present to the formal healthcare system. It is a challenge to use these data because of varying age ranges of reporting, different diagnosis techniques, surveillance methods, and healthcare utilization. This analysis examined data from 37 Demographic and Health Surveys and Malaria Indicator Surveys across 19 countries in sub-Saharan Africa collected between 2011 and 2018. The outcome of interest is a proxy indicator for severe malaria, defined as a proportion of children aged 6-59 months with at least one self-reported symptom of severe illness including loss of consciousness, rapid breathing, seizures, or severe anemia (hemoglobin $<5 \mathrm{~g} / \mathrm{dL}$ ) among those who were positive for malaria. The study includes a weighted descriptive, country-level analysis and a multilevel mixed-effects logistic regression model to assess the determinants of severe malaria. Among children positive for malaria across all surveys, $4.5 \%(95 \% \mathrm{Cl}: 4.1-4.8)$ had at least one sign or symptom of severe malaria, which was significantly associated with age, residence, wealth, and year of survey fieldwork at a $P$-value less than 0.05 . This analysis presents a novel and an alternative approach of estimating the fraction of severe malaria cases among malaria-positive children younger than 5 years in malaria-endemic countries. Estimating severe malaria cases through population-based surveys allows countries to estimate severe malaria across time and to compare with other countries. Having a population-level estimate of severe malaria cases helps further our understanding of the burden and epidemiology of severe malaria.
\end{abstract}

\section{INTRODUCTION}

In 2018 , an estimated 405,000 deaths from malaria occurred globally, with an estimated $70 \%$ of all malaria deaths occurring in children younger than 5 years in sub-Saharan Africa (SSA). ${ }^{1}$ Malaria typically begins as an acute febrile illness. If not appropriately treated, Plasmodium falciparum (P. falciparum) malaria can progress to severe illness and can often lead to death. Children with severe malaria frequently develop one or more of the following complications: severe anemia, respiratory distress, or cerebral malaria. ${ }^{2,3}$ The clinical manifestations of these severe malaria complications in children include impaired consciousness, respiratory distress (acidotic breathing), multiple convulsions, prostrations, shock, and jaundice. ${ }^{2,4}$ The clinical epidemiology of severe malaria can also present differently according to age and transmission intensity. Studies have shown that as the intensity of malaria transmission increases, the mean age of severe malaria decreases. ${ }^{5-8}$ In high-transmission areas, the risk for severe malaria is greatest among young children (the first few months of life to age 5 years), with severe anemia presenting as the most predominant complication. ${ }^{7,8}$ Severe malaria becomes less common in older children when acquired immunity provides a protective effect. Conversely, in low-transmission areas, severe malaria is more common in older children and adults, with cerebral malaria presenting as the predominant complication. ${ }^{7}$

Although the clinical features and transmission patterns of severe malaria are well understood, accurately capturing severe malaria cases among malaria positive cases at the population level remains a challenge. First, severe malaria symptoms are nonspecific, which makes it difficult to differentiate severe malaria cases from other diseases that are also

*Address correspondence to Cameron Taylor, The DHS Program, ICF, 530 Gaither Rd., Suite 500, Rockville, MD 20850. E-mail: cameron.taylor@icf.com common in malaria-endemic countries. ${ }^{2}$ Second, severe malaria case estimates from routine data collected through formal healthcare services are often affected by variable data quality and nonstandard case definitions, which make comparisons across countries challenging..$^{9-11}$ Finally, a proportion of severe malaria illnesses and deaths occurs outside the formal healthcare system, and is therefore undocumented. . $^{7,9,12-14}$

An accurate estimate of severe malaria among malaria-positive cases is essential to assess the impact of malaria prevention and control interventions and to guide future malaria investments. To date, the only robust estimates of severe malaria cases include children who present to the formal healthcare system or data from epidemiological studies coming from continuous surveillance systems (such as health and demographic surveillance systems [HDSS]) across SSA. It has been a challenge to use these data to model the fraction of severe malaria cases across SSA because of varying age ranges of reporting, different diagnosis techniques, surveillance methods, and healthcare utilization. 5,8 The Demographic and Health Surveys (DHS) Program, the primary global source of population-based malaria data, tests children for malaria and also measures hemoglobin concentrations to assess anemia status. For children who test positive for malaria, the DHS Program collects data on severe malaria symptoms for referral purposes during data collection. However, this information has not been used to estimate the fraction of severe cases of all the malaria-positive children. To provide an estimate of this fraction in SSA, this article presents an analysis of severe malaria signs and symptoms in children aged 6-59 months who are positive for $P$. falciparum malaria, and whose data are captured in population-based surveys.

\section{METHODS}

Data on severe malaria. This study used data from DHS and Malaria Indicator Surveys (MIS), which are both nationally representative, population-based household surveys of the DHS Program. ${ }^{15}$ All surveys are independent but use standardized data collection procedures and tools. When 
requested by the country, the DHS and MIS include the collection of blood samples for anemia and malaria testing among children aged 6-59 months. All children are tested regardless of whether they have signs or symptoms of malaria. Specially trained biomarker health technicians (who are usually nurses) take capillary blood obtained with a finger or heel prick. The blood is immediately tested for anemia and malaria in the field, and the results are provided to respondents' parents or guardians within 15-20 minutes.

In the field, biomarker health technicians use rapid diagnostic tests (RDTs) to determine if children have malaria. The RDTs detect the histidine-rich protein II (HRP-II) antigen of $P$. falciparum in blood. Plasmodium falciparum is the primary cause of severe malaria and is the predominant species found in the countries included in this analysis. ${ }^{2,7}$ Often, blood is also collected for the examination of blood smears by microscopy in the laboratory. Anemia is assessed by determining hemoglobin concentrations in the blood using the HemoCue $201+$ or, on occasion, the HemoCue 301 point-of-care hemoglobin testing system.

When the DHS and MIS first introduced malaria testing in 2006, biomarker health technicians offered antimalarial treatment to all children with positive RDT results. Beginning in 2011, the DHS Program instituted a referral system in which children who are positive for malaria but have no signs and symptoms of severe illness and are not anemic receive antimalarial treatment. Children who are positive for malaria and have signs and symptoms of severe illness or low hemoglobin concentrations are referred to the nearest health facility for treatment.

To accomplish this, additional questions were added to the survey's questionnaire to screen children for signs and symptoms of severe illness. To assess signs and symptoms of severe illness among RDT-positive children, the caregiver is asked: "Does (NAME) suffer from any of the following illnesses or symptoms: extreme weakness, heart problems, inability to drink or breastfeed, vomiting everything, loss of consciousness, rapid breathing, seizures, bleeding, jaundice, or dark urine?" If the child is positive for malaria (by RDT) and the caregiver answers "Yes" to any of the signs and symptoms and/or if the child has moderate-to-severe anemia defined as a hemoglobin concentration of $<8$ grams per deciliter $(\mathrm{g} / \mathrm{dL})$, the child is given a referral slip to take to the nearest healthcare facility for care. ${ }^{16}$ A child who is negative for malaria (by RDT) is not asked about severe illness symptoms (see Supplemental Figure 1 for more information about the DHS Program malaria referral process).

Data analysis. Case definition of severe malaria. This analysis used a severe malaria case definition based on signs and symptoms captured in DHS and MIS that most closely aligns with the clinical symptoms of malaria outlined in the WHO Management of Severe Malaria Handbook. ${ }^{2}$ Of the 11 signs and symptoms of severe illness collected by DHS and MIS, loss of consciousness, rapid breathing, seizures, or severe anemia (hemoglobin levels $<5 \mathrm{~g} / \mathrm{dL}$ adjusted for altitude) were the four clinical manifestations of severe illness in children that most closely align with the symptoms identified in the WHO Management of Severe Malaria Handbook for the clinical features of severe malaria. Hemoglobin levels are adjusted for the altitude in areas that are above 1,000 meters in elevation. ${ }^{17}$

Study variables. Outcome variable: Severe malaria. The outcome of interest is a proxy indicator of severe malaria, defined as the proportion of children aged 6-59 months with at least one symptom of severe illness of who those were positive for malaria, according to RDT. Symptoms of severe illness that were examined as a proxy for severe malaria included loss of consciousness, rapid breathing, seizures, or severe anemia (hemoglobin levels $<5 \mathrm{~g} / \mathrm{dL}$ adjusted for altitude).

Covariates: All potential confounders. For this analysis, variables found in the literature related to severe malaria were reviewed and included based on data availability. The model included the following covariates: gender, age, place of residence, wealth quintiles, malaria endemicity, year of survey fieldwork, and country (Table 1). The child's age was divided into four categories: 6-23 months, 24-35 months, 3647 months, and 48-59 months. Place of residence is defined as whether a household is located in a rural or urban area. Wealth quintiles were derived from the DHS wealth index, which measures the relative socioeconomic status of households based on household assets and amenities at the point in time of data collection. ${ }^{18}$ Year of survey fieldwork is divided into two equal categories based on the fieldwork years 2011-2014 and 2015-2018. To determine malaria endemicity, the authors assigned each malaria-positive child's household enumeration area into geographical zones based on malaria transmission risk. To link the DHS and MIS geo-coordinates (latitude and longitude) of each survey enumeration area to transmission risk zones, the authors used geo-coordinated $P$. falciparum parasite prevalence rates among children aged 2-10 years $\left(\mathrm{PfPR}_{2-10}\right)$ from the Malaria Atlas Project $2015 .^{19}$ Geographic coordinates for DHS and MIS clusters are randomly displaced to protect confidentiality, within a 2-km buffer for urban clusters and a 10-km buffer for rural clusters. To account for displacement, the mean of each pixel within the buffer was calculated for the PfPR ${ }_{2-10}$ values. The authors assigned every malaria-positive child's household in an enumeration area from the DHS or MIS dataset to the same malaria transmission risk zone based on corresponding $\mathrm{PfPR}_{2-10}$ data for that enumeration area. For the transmission zone categories, the authors used risk categories as outlined in the WHO Management of Severe Malaria Handbook, ${ }^{2}$ with low transmission defined as $P_{f P R_{2-10}} \leq 10 \%$ and high transmission defined as $P f R_{2-10}>50 \%$. Because of a high number of children in the moderate risk category $\left(11 \%<P f \mathrm{PR}_{2-10}\right.$ $\leq 50 \%$ ), the authors divided this risk group into two equal risk categories of moderate transmission A $\left(11 \%<\mathrm{PfPR}_{2-10}\right.$ $\leq 30 \%)$ and moderate transmission $B\left(31 \%<P f P R_{2-10} \leq 50 \%\right)$.

Study population. The inclusion criteria for the study were all malaria-endemic countries in SSA that have conducted a DHS or MIS in which children were tested for malaria and anemia, and included questions on signs or symptoms of severe illness. In total, this analysis examined results from 37 surveys across 19 countries (Figure 1). The study population for this analysis included malaria-positive children aged 659 months who stayed in surveyed households the night before the survey and received $P$. falciparum malaria parasite (RDT) and anemia tests. Children with any missing household enumeration area $\mathrm{PfPR}_{2-10}$ data were excluded from the analysis.

Regression analysis. The study includes a country-level descriptive analysis weighted for complex survey design and a multicountry weighted pooled analysis, both with $95 \%$ Cls. The study used a multilevel (individual-level and country-level) unweighted mixed-effects logistic regression model to assess the determinants of severe malaria among children with malaria infection. The model includes gender, age of the child, residence, household wealth, malaria transmission zones, and 
TABLE 1

Descriptions of covariates included in the analysis

\begin{tabular}{|c|c|c|c|}
\hline Variable & Type & Option & Details of measurement \\
\hline Gender & Categorical & Two categories: male and female & $\begin{array}{l}\text { Collected from the household } \\
\text { questionnaire }\end{array}$ \\
\hline Age-group & Categorical & $\begin{array}{l}\text { Four categories (months): 6-23, 24-35, } \\
36-47 \text {, and } 48-59\end{array}$ & $\begin{array}{l}\text { Based on date of birth and date of } \\
\text { interview from the household } \\
\text { questionnaire }\end{array}$ \\
\hline Place of residence & Categorical & Two categories: urban and rural & $\begin{array}{l}\text { Household classified as being in an urban } \\
\text { or rural area. Definition of urban and } \\
\text { rural is survey specific and is defined by } \\
\text { the country's statistical office }\end{array}$ \\
\hline Wealth quintile & Categorical & $\begin{array}{l}\text { Five categories: lowest, second, middle, } \\
\text { fourth, and highest }\end{array}$ & $\begin{array}{l}\text { Asset-based principal component } \\
\text { analysis }\end{array}$ \\
\hline Malaria endemicity & Categorical & $\begin{array}{l}\text { Four categories: low risk, intermediate } \\
\text { risk } A \text {, intermediate risk } B \text {, and high risk }\end{array}$ & $\begin{array}{l}\text { Categorized using Malaria Atlas Project } \\
\text { (MAP) } \text { PfPR }_{2-10} \text { values }\end{array}$ \\
\hline Fieldwork year & Categorical & $\begin{array}{l}\text { Two categories: } 2011-2014 \text { and } \\
\text { 2015-2018 }\end{array}$ & Based on the year of survey fieldwork \\
\hline Country & Categorical & $\begin{array}{l}19 \text { categories representing each of the } \\
\text { country included in the study }\end{array}$ & Based on survey country \\
\hline
\end{tabular}

year of survey fieldwork. All analyses were conducted with StataSE16 (StataCorp LP, College Station, TX).

\section{RESULTS}

Descriptive analysis. Country-level analysis. A total of 47,647 children aged 6-59 months were positive for malaria among the 183,265 children who met the inclusion criteria. Among children positive for malaria across all surveys, $4.5 \%$ (95\% Cl: 4.1-4.8) of children had at least one severe malaria symptom (loss of consciousness, seizures, rapid breathing, or severe anemia), ranging from $<0.1 \%$ in the Senegal DHS 2015 to $17.4 \%$ in the Madagascar MIS 2011. The prevalence of individual symptoms varies by country. Mali DHS 2018 (2.3\%)

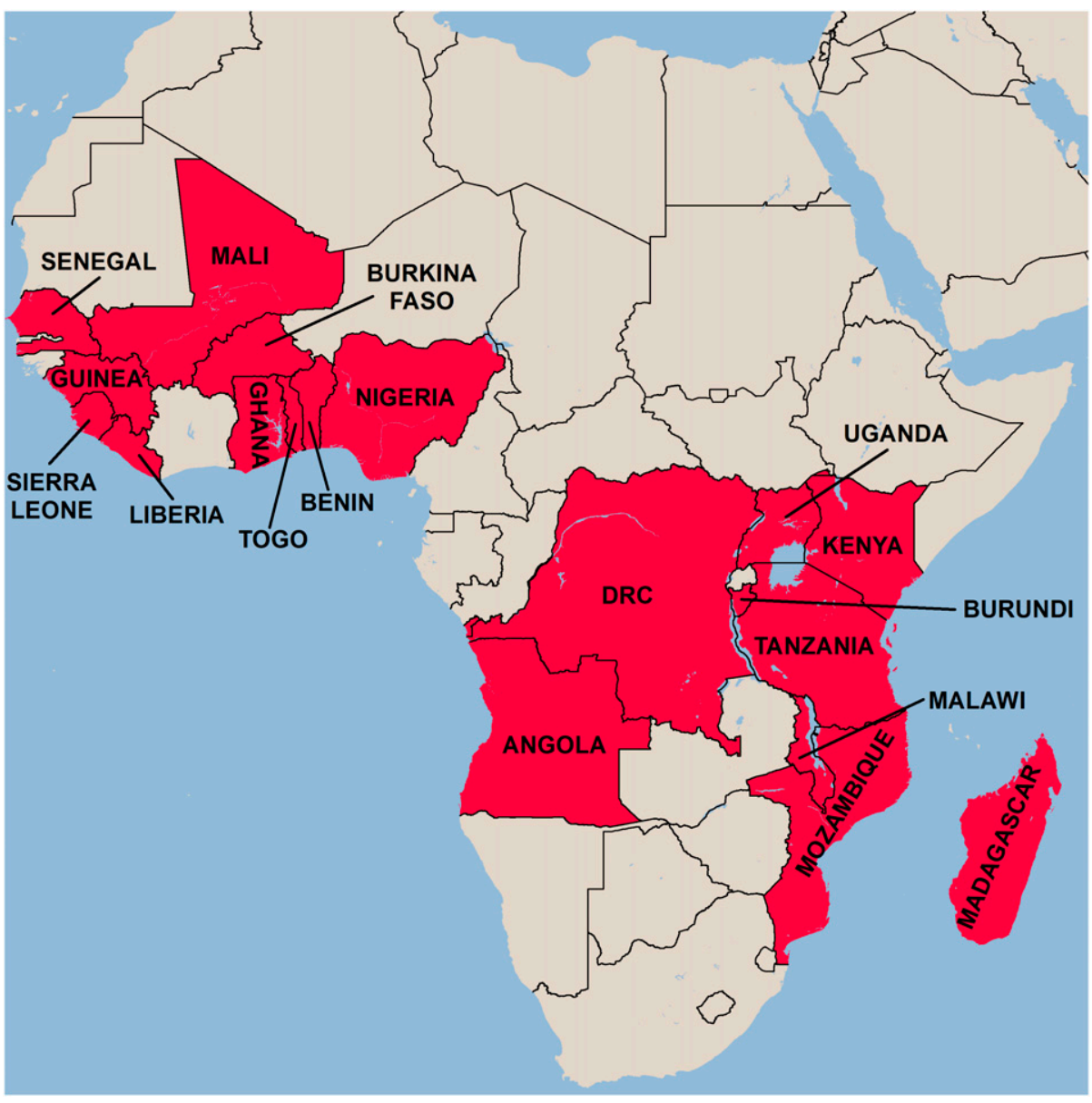

FiguRE 1. Map of countries included in the study. This figure appears in color at www.ajtmh.org. 
had the highest percentage of children with loss of consciousness, Guinea DHS 2012 (9.3\%) the highest percentage of children with seizures, Madagascar MIS 2011 (14.2\%) the highest percentage of children with rapid breathing, and Senegal DHS 2016 (3.2\%) the highest percentage of children with severe anemia among children who were positive for malaria via RDT (Table 2).

Multicountry pooled analysis. An examination of all children with at least one severe malaria symptom among children positive for malaria by background characteristics revealed nearly identical proportions by gender $(4.4 \%$ of males and $4.5 \%$ of females). The proportion of children with at least one severe malaria symptom decreased with age, ranging from $5.5 \%$ of children aged $6-23$ months $(95 \% \mathrm{Cl}: 4.9-6.0)$ to $3.3 \%$ of children aged $48-59$ months (95\% Cl: 2.9-3.8). Slightly more children symptomatic for severe malaria lived in urban areas (4.5\%, 95\% Cl: 4.2-4.9). In addition, a greater proportion of children from households in the lowest wealth quintile $(4.8 \%, 95 \% \mathrm{Cl}: 4.2-5.4)$ and in the highest transmission zone $(7.2 \%, 95 \% \mathrm{Cl}: 6.0-8.6)$ had at least one symptom of severe malaria compared with those in higher wealth quintiles and other transmission zones. A higher percentage of children $(6.1 \%, 95 \% \mathrm{Cl}$ : 49.8-57.9) with at least one severe malaria symptom were surveyed between 2011 and 2014 as compared with children surveyed between 2015 and 2018 (Table 3).

Regression analysis. The regression analysis shows the adjusted odds of reporting at least one symptom of severe malaria in relation to the different background characteristics of the children. This regression is adjusted for gender, age of the child, residence, household wealth, malaria transmission zone, and year of survey fieldwork. Compared with children aged 6-23 months, children aged 36-47 months (Adjusted odds ratio [AOR]: 0.73; 95\% Cl: 0.65-0.82) and 48-59 months (AOR: $0.58 ; 95 \% \mathrm{Cl}: 0.51-0.66$ ) were significantly less likely to report at least one symptom of severe malaria. Urban children were significantly more likely to report at least one symptom of severe malaria than rural children (AOR: $1.28 ; 95 \% \mathrm{Cl}$ : 1.12-1.48). Socioeconomic status of the household is associated with the likelihood of reporting a severe malaria symptom, with children in the highest wealth quintile having lower odds than those from households in the lowest quintile (AOR: 0.56; 95\% Cl: 0.42-0.76). Malaria endemicity defined by different transmission zones was not associated with the likelihood of reporting a symptom of severe malaria. Finally, the children surveyed between 2015 and 2018 were significantly less likely to report at least one symptom of severe malaria than those surveyed between 2011 and 2014 (AOR: 0.72; 95\% Cl: 0.62-0.83; Table 4).

\section{DISCUSSION}

Despite improvements in the diagnosis and documentation of severe malaria cases, there remains uncertainty about the fraction of malaria cases that progress to severe infection at the population level. The authors present an estimate of the fraction of severe malaria cases among children with malaria infection from 19 malaria-endemic countries in SSA. Our estimates are based on data from population-based household surveys that allow severe malaria cases outside of the formal healthcare system to be directly captured in estimates. Estimating the fraction of severe malaria out of malaria-infected children through household surveys provides countries with a standardized estimate comparable across time as well with other countries. Routine data collected at health facilities should still be considered the gold standard for estimating severe malaria due to the examination of the child by a trained medical provider. Household survey data coupled with severe malaria case data from routine health facility records will help strengthen our overall knowledge of the burden of severe malaria at the population level.

In this study, $4.5 \%$ of all malaria-infected children reported at least one symptom of severe malaria, which is similar with other estimates of severe malaria. ${ }^{1}$ This acknowledges that other estimates of severe malaria cases account only for malaria-infected children who access the formal healthcare system. ${ }^{1,12}$ Household surveys test all children aged 659 months for malaria, and whereas some of the children who were showing signs of severe illness would have eventually accessed the formal healthcare system, some of these children would have died, recovered at home, or received care outside of the formal healthcare system. ${ }^{7,9,12-14}$ Although there is a large range in severe malaria across the 19 countries included in this analysis, this can be driven by low numbers of malaria-positive children in some countries.

Findings from this study confirm previous observations that severe malaria is dependent on age and transmission intensity. ${ }^{5-8}$ Younger children were significantly more likely to have severe malaria, and although not significant, the risk of severe malaria was greater in high malaria transmission zones. However, unlike previous research, this study did not find an interaction between age and the intensity of malaria transmission in relation to having at least one symptom of severe malaria (data not shown). ${ }^{5}$ One explanation is the limited age range of children (6-59 months) in this analysis. Past studies that have examined the association of variations in age and endemicity on the clinical manifestation of severe malaria included children up to the age of 10 years. ${ }^{5,6,8}$

Malaria-infected children surveyed between 2015 and 2018 were significantly less likely to have severe malaria symptoms than those surveyed between 2011 and 2014. This is controlling for malaria transmission level. This finding aligns with the 2019 World Malaria Report, which reported a decrease in malaria deaths since 2010 . $^{1}$ Moreover, prompt diagnosis and treatment-seeking rates have generally increased, helping to prevent mild cases of malaria from developing into severe illness and death. 2,20

This analysis also indirectly highlights potential variation in care-seeking patterns for severe malaria cases across SSA. Urban malaria-infected children were significantly more likely to have at least one severe malaria symptom than rural children. This could be due to urban caregivers having access to private health facilities, pharmacy shops, and other medical vendors that are more prevalent in urban communities. ${ }^{21}$ These types of healthcare service providers commonly practice presumptive malaria treatment or prescribe unrecommended drugs. ${ }^{22-24}$ Other reasons could be attributed to self-treatment at home..$^{23,24}$ However, more exploration is needed into these patterns. In addition, the country was highly significant in the model. By examining the fraction of severe malaria cases among malaria-infected children at the household level, this analysis potentially includes children whose caregivers have taken them to a healthcare facility but whose illness did not resolve or have not sought care for the child's 


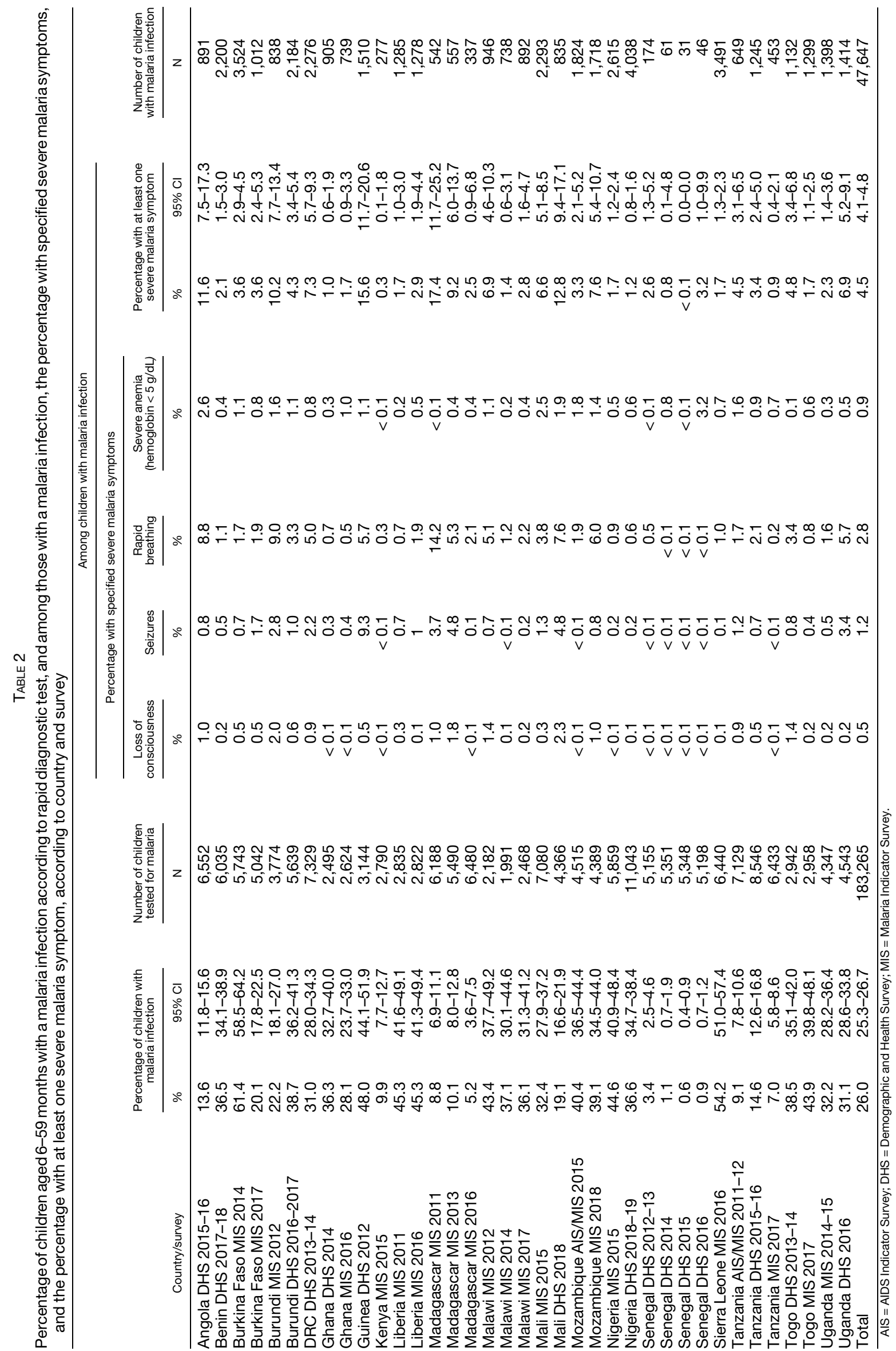


TABLE 3

Percentage of children who had at least one severe malaria symptom among children who were positive for malaria, pooled weighted analysis

\begin{tabular}{|c|c|c|c|}
\hline Background characteristics & Percentage & $95 \%$ Cls & Number of children with a malaria infection \\
\hline \multicolumn{4}{|l|}{ Child } \\
\hline \multicolumn{4}{|l|}{ Gender } \\
\hline Male & 4.4 & $4.0-4.8$ & 24,294 \\
\hline Female & 4.5 & $4.1-5.0$ & 23,353 \\
\hline \multicolumn{4}{|l|}{ Age-group (months) } \\
\hline $6-23$ & 5.5 & $4.9-6.0$ & 12,262 \\
\hline $24-35$ & 5.1 & $4.5-5.7$ & 10,669 \\
\hline $36-47$ & 4.0 & $3.6-4.5$ & 12,098 \\
\hline $48-59$ & 3.3 & $2.9-3.8$ & 12,619 \\
\hline \multicolumn{4}{|l|}{ Household } \\
\hline \multicolumn{4}{|l|}{ Place of residence } \\
\hline Rural & 4.1 & $3.4-4.9$ & 40,818 \\
\hline Urban & 4.5 & $4.2-4.9$ & 6,829 \\
\hline \multicolumn{4}{|l|}{ Wealth quintile } \\
\hline Lowest & 4.8 & $4.2-5.4$ & 15,302 \\
\hline Second & 4.5 & $4.0-5.0$ & 13,510 \\
\hline Middle & 4.2 & $3.7-4.8$ & 10,254 \\
\hline Fourth & 4.7 & $4.0-5.4$ & 6,518 \\
\hline Highest & 2.6 & $1.9-3.7$ & 2,063 \\
\hline \multicolumn{4}{|l|}{ Malaria endemicity } \\
\hline Low transmission $<10 \%$ & 5.5 & $4.6-6.6$ & 5,507 \\
\hline Moderate transmission A $11-30 \%$ & 4.3 & $3.8-4.8$ & 18,531 \\
\hline Moderate transmission B $31-50 \%$ & 3.4 & $3.0-3.9$ & 17,760 \\
\hline High transmission > 50\% & 7.2 & $6.0-8.6$ & 5,849 \\
\hline \multicolumn{4}{|l|}{ Fieldwork year } \\
\hline 2011-2014 & 6.1 & $5.4-6.9$ & 15,977 \\
\hline 2015-2018 & 3.6 & $3.3-4.0$ & 31,670 \\
\hline Total & 4.5 & $4.1-4.8$ & 47,647 \\
\hline
\end{tabular}

illness. Although the decision to seek care is ultimately made by the caregiver, it is highly influenced by factors such as the availability of government-based facilities, country wealth, cost of care, and education. ${ }^{14,20,25-27}$ Further exploration is

TABLE 4

Multilevel unweighted mixed-effects logistic regression model of children who had at least one symptom of severe malaria among children who were positive for malaria, pooled analysis

\begin{tabular}{lcc}
\hline \multicolumn{1}{c}{ Background characteristic } & Adjusted odds ratio & $95 \% \mathrm{Cls}$ \\
\hline Child & & \\
Gender & & \\
Male & 1 (reference) & \\
Female & 0.95 & $0.87-1.04$ \\
Age-group (months) & & \\
$6-23$ & 1 (reference) & \\
$24-35$ & 0.91 & $0.81-1.02$ \\
36-47 & $0.73^{\star \star \star}$ & $0.65-0.82$ \\
48-59 & $0.58^{\star \star \star}$ & $0.51-0.66$ \\
Household & & \\
Place of residence & & \\
Rural & 1 (reference) & \\
Urban & $1.28^{\star \star \star}$ & $1.12-1.48$ \\
Wealth quintile & & \\
Lowest & 1 (reference) & \\
Second & 1.01 & $0.90-1.13$ \\
Middle & 0.91 & $0.80-1.03$ \\
Fourth & 0.99 & $0.85-1.14$ \\
Highest & $0.56^{\star \star \star}$ & $0.42-0.76$ \\
Malaria endemicity & 1 (reference) & \\
Low transmission $<10 \%$ & 1.01 & $0.86-1.19$ \\
Moderate transmission A 11-30\% & 0.99 & $0.81-1.22$ \\
Moderate transmission B 31-50\% & 1.06 & $0.83-1.34$ \\
High transmission $>50 \%$ & & \\
Fieldwork year & & \\
2011-2014 & (reference) & \\
2015-2018 & $0.72^{\star \star \star}$ & $0.62-0.83$ \\
\hline
\end{tabular}

needed into country and urban-rural variations in care-seeking and its association with severe malaria burden estimates.

This study has several limitations. Children with severe malaria frequently develop one or more complications, including severe anemia, respiratory distress, or cerebral malaria. This analysis examined children who had at least one symptom of severe malaria. The authors did not disaggregate this analysis by proxies for respiratory distress or cerebral malaria. Examining severe anemia is possible because this is a discrete diagnosis based on hemoglobin levels. However, to fully disaggregate by respiratory distress or cerebral malaria would require additional questions about symptoms such as prostration and the number and severity of convulsions. ${ }^{2}$

The use of household-level data is a noteworthy advantage of this study, but it also introduces a principal limitation. There is a risk of including uncomplicated malaria cases or nonmalaria cases in our proxy definition. Malaria positivity is based on RDT-detectable antigens that continue to circulate in the blood after the infection has cleared, and severe malaria symptoms are nonspecific. ${ }^{28}$ Our definition of severe malaria relies on caregiver report rather than a diagnosis by a clinician at a health facility. Although the interviewer for the biomarker questionnaire is a trained biomarker health technician (usually a nurse), which may improve the questionnaire responses, the nonspecific nature of severe malaria remains an issue. The authors have addressed this limitation by narrowing the proxy definition of severe malaria to only examine loss of consciousness, rapid breathing, seizures, or severe anemia (hemoglobin $<5 \mathrm{~g} / \mathrm{dL}$ adjusted for altitude). These symptoms are more distinct than some other symptoms (extreme weakness and heart problems) caregivers are asked and most closely align with the WHO clinical manifestations of severe malaria. 
The authors assumed these symptoms would not be confused by caregivers, even those with a limited education. However, both cultural and regional differences in caregivers' understanding and reporting of symptoms could exist. This study was unable to examine the reliability of reported signs and symptoms because caregivers were not asked questions on severe malaria symptoms for malaria-negative children. In addition, by limiting our definition of severe malaria symptoms, there is the possibility that we may be missing cases.

This analysis only includes children with malaria according to RDT, which further minimizes the possibility that the child is sick with an illness other than severe malaria. More sensitive measures of malaria diagnosis than standard HRP-II RDTs should be explored, such as microscopy, highly sensitive RDTs, or PCR.

\section{CONCLUSION}

This analysis investigated severe malaria symptoms in children aged 6-59 months who are positive for malaria as identified in population-based surveys. To date, there has been a gap in knowledge about the fraction of severe malaria out of malaria-infected case at the population level because previous estimates have relied exclusively on data from formal healthcare services. This analysis presents an estimate of fraction of severe malaria cases among children aged 659 months infected with malaria from 19 countries across multiple malaria endemicity zones. The data in this analysis were initially collected for severe illness referral purposes, but also provide invaluable insights into severe malaria cases at the population level.

Received October 14, 2020. Accepted for publication December 18, 2020.

Published online February 1, 2021.

Note: Supplemental figure appears at www.ajtmh.org.

Acknowledgments: We would like to thank Tom Fish, the DHS Program geospatial analyst, for his help in preparing the $P f \mathrm{PR}_{2-10}$ data for merging as well as the creation of the analysis map. We would also like to thank Diane Stoy for editorial review and Annette McFarland for assisting in the creation of graphics. In addition, we thank Jordan Burns, Misun Choi, Lia Florey, Kevin Griffith, Kathryn Hogan, and Meera Venkatesan of PMI at USAID for their invaluable feedback on this analysis. We would like to thank Bernard Barrère (1953-2018), who diligently served the DHS Program for 29 years in various capacities. Bernard was the impetus for the inclusion of severe malaria questions in 2011 as part of the severe malaria referral process. We hope that this analysis extends the impact that Bernard had on the world through his life of service to global health and data.

Financial support: Cameron Taylor, Sorrel Namaste, Joanna Lowell, and Johanna Useem were supported by the U.S. Agency for International Development (USAID) through the DHS Program (\#720OAA-18C-00083). Yazoume Ye was supported through the USAID and PMI under the terms of the PMI Measure Malaria Associate Award No. 7200AA19LA00001. PMI Measure Malaria is implemented by the University of North Carolina at Chapel Hill, in partnership with ICF Macro, Inc.; Tulane University; John Snow, Inc.; and Palladium International, LLC.

Disclosure: C. T., S. M. L. N., and J. L. conceived of and designed the study. C. T. and J. U. assisted in the collection of study variables. C. T. performed all data analysis, with S. M. L. N. and Y. Y. contributing to the interpretation. C. T. wrote the manuscript with inputs from S. M. L. N, J. L., J. U., and Y. Y. All authors read and approved the final manuscript.

Authors' addresses: Cameron Taylor, Sorrel M. L. Namaste, and Johanna Useem, The DHS Program, ICF, Rockville, MD, E-mails: cameron.taylor@icf.com, sorrel.namaste@icf.com, and hanna.useem@ icf.com. Joanna Lowell, The DHS Program, Vysnova Partners, Landover, MD, E-mail: joanna.lowell@icf.com. Yazoumé Yé, PMI Measure Malaria, ICF, Rockville, MD, E-mail: yazoume.ye@icf.com.

This is an open-access article distributed under the terms of the Creative Commons Attribution (CC-BY) License, which permits unrestricted use, distribution, and reproduction in any medium, provided the original author and source are credited.

\section{REFERENCES}

1. WHO, 2019. World Malaria Report. Geneva, Switzerland: World Health Organization.

2. WHO, 2013. Management of Severe Malaria - A Practical Handbook. Third edition. Geneva, Switzerland: World Health Organization.

3. WHO, 2019. Malaria Fact Sheet. Geneva, Switzerland: World Health Organization. Available at: https://www.who.int/newsroom/fact-sheets/detail/malaria. Accessed July 22, 2020.

4. Bejon $P$ et al., 2007. Defining childhood severe falciparum malaria for intervention studies. PLOS Med 4: e251.

5. Okiro EA, Al-Taiar A, Reyburn H, Idro R, Berkley JA, Snow RW, 2009. Age patterns of severe paediatric malaria and their relationship to Plasmodium falciparum transmission intensity. Malar J 8: 4.

6. Carneiro I, Roca-Feltrer A, Griffin JT, Smith L, Tanner M, Schellenberg JA, Greenwood B, Schellenberg D, 2010. Agepatterns of malaria vary with severity, transmission intensity and seasonality in sub-Saharan Africa: a systematic review and pooled analysis. PLoS One 5: e8988.

7. WHO, 2014. Severe malaria. Trop Med Int Health 19 (Suppl 1): 7-131.

8. Roca-Feltrer A, Carneiro I, Smith L, Schellenberg JRMA, Greenwood B, Schellenberg D, 2010. The age patterns of severe malaria syndromes in sub-Saharan Africa across a range of transmission intensities and seasonality settings. Malar J 9: 282.

9. Camponovo F, Bever CA, Galactionova K, Smith T, Penny MA, 2017. Incidence and admission rates for severe malaria and their impact on mortality in Africa. Malar J 16: 1.

10. Ye Y, Kyobutungi C, Ogutu B, Villegas L, Diallo D, Tinto H, Oduro A, Sankoh O, 2013. Malaria mortality estimates: need for agreeable approach. Trop Med Int Health 18: 219-221.

11. Mathers CD, Fat DM, Inoue M, Rao C, Lopez AD, 2005. Counting the dead and what they died from: an assessment of the global status of cause of death data. Bull World Health Organ 83: 171-177.

12. Roca-Feltrer A, Carneiro I, Armstrong Schellenberg JR, 2008. Estimates of the burden of malaria morbidity in Africa in children under the age of 5 years. Trop Med Int Health 13: 771-783.

13. Reyburn $\mathrm{H}$ et al., 2005. Association of transmission intensity and age with clinical manifestations and case fatality of severe Plasmodium falciparum malaria. JAMA 293: 1461-1470.

14. Alegana VA, Maina J, Ouma PO, Macharia PM, Wright J, Atkinson PM, Okiro EA, Snow RW, Tatem AJ, 2018. National and subnational variation in patterns of febrile case management in sub-Saharan Africa. Nat Commun 9: 4994.

15. The Demographic and Health Surveys (DHS) Program, 2020. Survey Types. Available at: https://dhsprogram.com/WhatWe-Do/Survey-Types/index.cfm. Accessed January 2, 2020.

16. Malaria. RPtE, 2016. Malaria Indicator Survey Toolkit. Available at: https://www.malariasurveys.org/toolkit.cfm. Accessed March 9, 2020.

17. Croft TN, Marshall AMJ, CK A, 2018. Guide to DHS Statistics. Rockville, MD: ICF.

18. Rutstein SO, Johnson K, 2004. The DHS Wealth Index. Calverton, Maryland: ORC Macro.

19. Pfeffer DA et al., 2018. malariaAtlas: an $R$ interface to global malariometric data hosted by the Malaria Atlas Project. Malar $J$ 17: 352.

20. Battle KE et al., 2016. Treatment-seeking rates in malaria endemic countries. Malar J 15: 20.

21. Maina J, Ouma PO, Macharia PM, Alegana VA, Mitto B, Fall IS, Noor AM, Snow RW, Okiro EA, 2019. A spatial database of 
health facilities managed by the public health sector in sub Saharan Africa. Sci Data 6: 134.

22. Awuah RB, Asante PY, Sakyi L, Biney AAE, Kushitor MK, Agyei F, de-Graft Aikins A, 2018. Factors associated with treatmentseeking for malaria in urban poor communities in Accra, Ghana. Malar J 17: 168.

23. Babalola OJ, Ajumobi O, Ajayi IO, 2020. Rural-urban disparities and factors associated with delayed care-seeking and testing for malaria before medication use by mothers of under-five children, Igabi LGA, Kaduna Nigeria. Malar J 19: 294.

24. Chuma J, Okungu V, Molyneux C, 2010. Barriers to prompt and effective malaria treatment among the poorest population in Kenya. Malar J 9: 144.

25. El-Houderi A, Constantin J, Castelnuovo E, Sauboin C, 2019. Economic and resource use associated with management of malaria in children aged $<5$ years in sub-Saharan Africa: a systematic literature review. MDM Policy Pract 4: 2381468319893986.

26. Issifou $S$, Kendjo E, Missinou M, Matsiegui $P$, Dzeing-Ella $A$, Dissanami F, Kombila M, Krishna S, Kremsner P, 2007. Differences in presentation of severe malaria in urban and rural Gabon. Am J Trop Med Hyg 77: 1015-1019.

27. Sumbele IUN, Kimbi HK, Ndamukong-Nyanga JL, Nweboh $M$, Anchang-Kimbi JK, Lum E, Nana Y, Ndamukong KKJ, Lehman LG, 2015. Malarial anaemia and anaemia severity in apparently healthy primary school children in urban and rural settings in the mount Cameroon area: cross sectional survey. PLoS One 10: e0123549.

28. Dalrymple U, Arambepola R, Gething PW, Cameron E, 2018. How long do rapid diagnostic tests remain positive after anti-malarial treatment? Malar J 17: 228. 